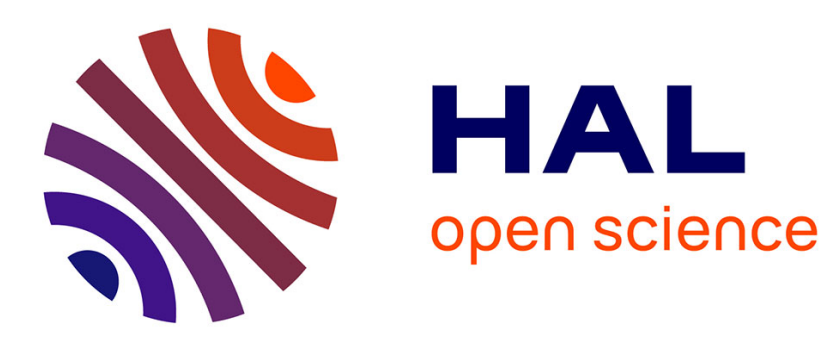

\title{
Wireless Passive Sensors Interrogation Technique Based on a Three-Dimensional Analysis
}

Dominique Henry, Hervé Aubert, Patrick Pons

\section{To cite this version:}

Dominique Henry, Hervé Aubert, Patrick Pons. Wireless Passive Sensors Interrogation Technique Based on a Three-Dimensional Analysis. European Microwave Week (EuMW), Oct 2016, London, United Kingdom. hal-01396848

\section{HAL Id: hal-01396848 \\ https://hal.science/hal-01396848}

Submitted on 15 Nov 2016

HAL is a multi-disciplinary open access archive for the deposit and dissemination of scientific research documents, whether they are published or not. The documents may come from teaching and research institutions in France or abroad, or from public or private research centers.
L'archive ouverte pluridisciplinaire HAL, est destinée au dépôt et à la diffusion de documents scientifiques de niveau recherche, publiés ou non, émanant des établissements d'enseignement et de recherche français ou étrangers, des laboratoires publics ou privés. 


\title{
Wireless Passive Sensors Interrogation Technique Based on a Three-Dimensional Analysis
}

\author{
D.Henry, H.Aubert and P.Pons \\ LAAS-CNRS ; 7 av. du colonel Roche \\ 31077 Toulouse, France \\ University of Toulouse; INP; \\ F-31077 Toulouse, France \\ dhenry@laas.fr, aubert@laas.fr,ppons@laas.fr
}

\begin{abstract}
This paper describes a novel wireless passive sensors interrogation technique based on a three-dimensional analysis. Variable impedances acting as passive sensors are remotely read by a $24 \mathrm{GHz}$ bistatic FMCW radar at distances of 2.0 meters and 5.5 meters. The radar performs a mechanical beam scanning to receive the electromagnetic echo level response in a volume. Several estimators are compared to calibrate and quantify the passive sensor input impedance. From this impedance the physical quantity of interest (temperature, pressure, strain ...) can be derived.
\end{abstract}

Keywords - wireless passive sensors, $3 D$ radar imaging, remote sensing, isosurfaces.

\section{INTRODUCTION}

The wireless interrogation of passive sensors (tags without batteries and integrated circuits) is challenging. Many applications require autonomous sensors with a long life-time able to measure various physical quantities such as temperature, pressure or moisture. However these sensors must achieve good performances in terms of distance of interrogation, size, resolution, sensitivity and full-scale measurement range. Radio Frequency IDentification (RFID) is the most commonly used technology for passive sensing. Sensing elements can be easily integrated in RFID tags but the distance of interrogation is limited to few meters due to power regulations. Surface Acoustic Wave (SAW) sensors are also investigated but their operating frequency is limited to few $\mathrm{GHz}$. Harmonic (or intermodulation) radars can remotely read tags with a good achievable range. Nevertheless their different carrier frequencies at transmission and reception do not fit with radio regulations.

A novel passive sensor interrogation technique based on a three-dimensional scanning has been recently reported by the authors in [1]. A Frequency-Modulated Continuous-Wave (FMCW) radar operating at $24 \mathrm{GHz}$ was used for the detection and localization of the sensors and for deriving the variation of sensors input impedance from standard algorithms and signal processing techniques applied to 3D radar images [1][2]. From this impedance the physical quantity of interest (temperature, pressure, strain ...) can be deduced. The present paper compares the performances of several possible estimators for the remote derivation of passive sensors input impedance from the three-dimensional analysis of radar images.

Section II describes the beam scanning radar system and the interrogated passive sensors. Section III defines various possible estimators for deriving the input impedance of the sensors from the $3 \mathrm{D}$ analysis of radar images. The impedance variation is finally displayed in three dimensions using isosurfaces.

\section{3D BEAM SCANNING TECHNIQUE}

\section{A. Reader system}

The reader is a bistatic FMCW radar transmitting a chirp at a carrier frequency $f_{c}=23.8 \mathrm{GHz}$ and frequency modulated with a triangular signal. This signal has a ramp-time $T=1 \mathrm{~ms}$ and a frequency bandwidth $B=2 \mathrm{GHz}$. The standard definition of the theoretical depth resolution $d$ is the given by [3]:

$$
d=\frac{c}{2 \cdot B}
$$

where $c$ is the celerity of light in air. In our configuration, a theoretical depth resolution of $7.5 \mathrm{~cm}$ can be reached. Different antennas are used for transmission and reception purposes. A parabolic antenna is connected to the front-end of the Tx channel. It has a gain of $33.5 \mathrm{dBi}$ and a beamwitdh of $2^{\circ}$. Knowing that the input power of the radar is $10.8 \mathrm{dBm}$, the EIRP is $44.3 \mathrm{dBm}$. The reception antenna is a $1 \times 5$ patches array with a gain of $8.6 \mathrm{dBi}$ and a beamwitdh of $80^{\circ}$ in azimuth and $20^{\circ}$ in elevation. The radar and its antennas are mounted on a pan-tilt platform automatically controlled by a computer unit (Figure 1). It performs a 3D spherical scanning from $\theta_{\text {Min }}=-10^{\circ}$ to $\theta_{\operatorname{Max}}=10^{\circ}$ in azimuth with a step $\Delta \theta=2^{\circ}$ and $\varphi_{\operatorname{Min}}=-10^{\circ}$ to $\varphi_{\text {Max }}=10^{\circ}$ in elevation with a step $\Delta \varphi=2^{\circ}$. For each step, a chirp is transmitted. The signal backscattered by the scene is then demodulated and filtered by the radar.

The passive sensor is composed of three parts : (a) a horn antenna of gain $20 \mathrm{dBi}$ and a $60^{\circ}$ beamwidth, (b) a coaxial cable with an effective length of $L=1.35 \mathrm{~m}$ (delay line) and (c) a variable attenuator terminated with a short-circuit. The measurement principle is based on load scatterers theory [4]. The structural mode is used to localize the sensor, and the sensing (or antenna) mode is analyze to derive the input impedance of the sensor and consequently, to deduce the physical quantity of interest. The variation of the sensor input impedance is simulated here from the tuning of an attenuator. 


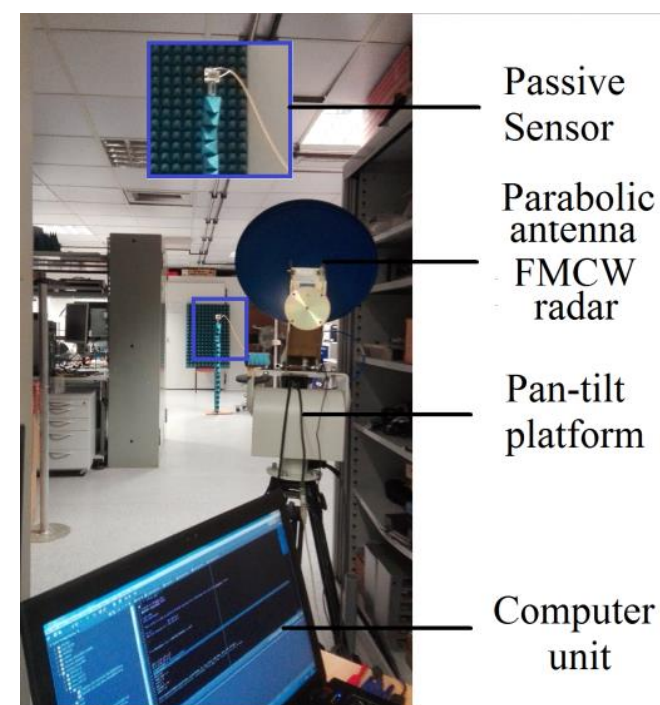

Fig 1. Measurement system and passive sensor located at 5.5 meters

\section{B. Data acquisition and signal processing}

For each sweep step we perform an averaging of 10 time signals. I and Q signals of the reception channel are used in order to compute the complex signal. The time signal is digitalized into 1024 samples and normalized with the maximal voltage of the ADC $(3.3 \mathrm{~V})$. A back-projection algorithm is applied to determine the echo level for each cell that has been scanned [5]. The rotating beam scanning generates resolution cells in spherical coordinates. The volume $V_{S p h e r}$ of such resolution cell is then given as follows:

$$
V_{\text {Spher }}=\frac{2}{3}\left[\left(R+\frac{d}{2}\right)^{3}-\left(R-\frac{d}{2}\right)^{3}\right] \cdot \Delta \theta \cdot \sin \left(\frac{\Delta \varphi}{2}\right)
$$

where $R$ denotes to the distance from the center of the resolution cell to the radar. The cell volume grows with the distance of interrogation. A transformation to Cartesian coordinates is applied with a spline interpolation for increasing the resolution and for facilitating the analysis of 3D radar image. The new volume $V_{\text {Cart }}$ of resolution cell and the Cartesian grid are defined such as:

$$
\begin{aligned}
& V_{\text {Cart }}=\Delta x \cdot \Delta y \cdot \Delta z \\
& \text { with }\left\{\begin{array}{c}
\Delta x=\frac{2 R_{\text {Min }}}{p_{x}} \tan \left(\frac{\Delta \theta}{2}\right) \\
\Delta y=\frac{2 R_{\text {Min }}}{p_{y}} \tan \left(\frac{\Delta \varphi}{2}\right) \\
\Delta z=\frac{c}{2 B \cdot p}=\frac{d}{p}
\end{array}\right.
\end{aligned}
$$

where $R_{\text {Min }}$ and $R_{\text {Max }}$ designate the minimal and maximal ranges in which the Cartesian transformation operates. The parameters $p_{x}$ and $p_{y}$ are increasing resolution factors of $\mathrm{x}$ and $\mathrm{y}$ cross-range axes. The parameter $p$ is a pad factor which represents a zoom range performed by the chirp-z transformation defines as follows [6]

$$
\begin{gathered}
\hat{s}=\sum_{n=0}^{N-1} s(n) \cdot A^{-n} \cdot W^{n \cdot k} \\
\text { with }\left\{\begin{array}{l}
A=e^{(-j \cdot 2 \cdot d k \cdot R)} \\
W=e^{(j \cdot 2 \cdot d k \cdot p)}
\end{array}\right.
\end{gathered}
$$

In Eq.(5) $\hat{s}$ denotes the result of the chirp-z transformation of the sampled time signal $s$ and $N$ is the total number of samples. In Eq.(6) $R$ designates the distance from the resolution cell to the radar and $d k$ is the wave number increment. The dimensions of the 3D Cartesian grid $\left(X_{M i n}\right.$, $\left.X_{\text {Max }}\right),\left(Y_{\text {Min }}, Y_{\text {Max }}\right)$ and $\left(Z_{\text {Min }}, Z_{\text {Max }}\right)$ are given by:

$$
\left\{\begin{array}{c}
\left(X_{\text {Min }}, X_{\text {Max }}\right)=\left(R_{\text {Min }} \tan \left(\theta_{\text {Min }}\right), R_{\text {Min }} \tan \left(\theta_{\text {Max }}\right)\right) \\
\left(Y_{\text {Min }}, Y_{\text {Max }}\right)=\left(R_{\text {Min }} \tan \left(\varphi_{\text {Min }}\right), R_{\text {Min }} \tan \left(\varphi_{\text {Max }}\right)\right) \\
\left(Z_{\text {Min }}, Z_{\text {Max }}\right)=\left(R_{\text {Min }}, \sqrt{R_{\text {Max }}{ }^{2}-X_{\text {Max }}{ }^{2}-Y_{\text {Max }}{ }^{2}}\right)
\end{array}\right.
$$

The Fig. 2 illustrates the Cartesian transformation Eqs. (3)-(6). The final step consists of displaying the data in three dimensions. This can be achieved with the help of isosurfaces given a representation of each layer of equal echo level (see section III).

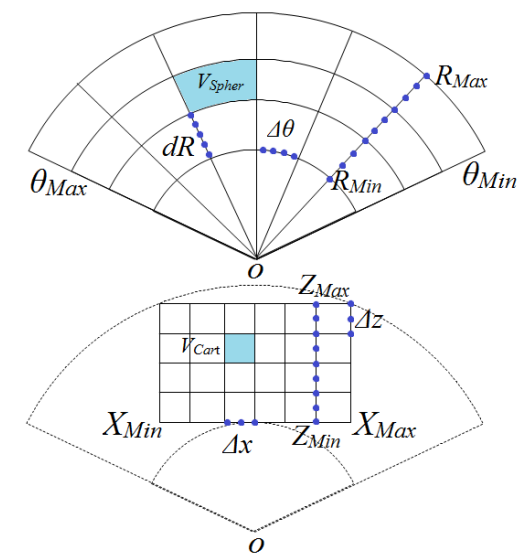

Fig 2. Scanning grid in spherical coordinates and its corresponding Cartesian grid after Cartesian transformation Eqs. (3)-(6) for a given elevation cut-plane

\section{MEASUREMENT RESULTS AND DISCUSSION}

Measurements are performed for different values of sensor impedances when the passive sensor is placed at a distance of 2 meters (scenario I) or 5.5 meters (scenario II) from the reader. Fig 2 shows the resulting 3D isosurfaces display for a sensor impedance of $0 \Omega$. Structural and sensing modes are clearly detectable and separated by the coaxial cable effective length (1.35 meters). In such isosurfaces echo levels having the same amplitudes are assigned to a specific colored layer. The transparency of each layer increases when the amplitude of the radar echo decreases. This visualization based on isosurfaces is a great tool for displaying a global volume and for roughly observing the 3D distribution of radar echo levels.

When the sensing mode is localized, a volume of study is defined around the corresponding radar echo level. This 
volume is defined in Table I. For the two above-mentioned scenarios, this volume is composed of several resolution cells, respectively 32832 cells (scenario I) or 23598 cells (scenario II). The volume is of $0.29 \mathrm{~m}^{3}$ (scenario I) and of $0.21 \mathrm{~m}^{3}$ (scenario II). Consequently the beam scanning analysis does not only focus on a single value of the magnitude, but on several values distributed in a three-dimensional space. This approach allows the definition of several estimators. These estimators may provide a direct relationship between the value of the sensor input impedance value and the magnitude variation of the sensing mode in a volume.

Fig.4.a shows the distribution of the echo level for a sensor input impedance of $50 \Omega$. By choosing a volume containing a sufficient number of resolution cells, the distribution tends to a log-normal distribution given by:

$$
\phi(x)=\frac{K}{x \sigma \sqrt{2 \pi}} e^{\left[-\frac{(\ln (x)-\mu)^{2}}{2 \sigma^{2}}\right]}
$$

where $K$ and $\sigma$ are scale factors and $\mu$ is the location factor. Each black bar corresponds to the weight of cells in which the echo level ranges between two extreme values defined by an echo level step (a step of $1 \mathrm{~dB}$ is chosen here for illustration purpose). The red curve is plotted by using a curve fitting algorithm with a cubic interpolation of 1000 points and parameters of the log-normal distribution in equation (8) are extracted.

Fig.4.b shows that the shape of the log-normal distribution changes with the sensor input impedance. Hence it is possible to define estimators which depend directly from the lognormal distribution parameters. In order to estimate the variation of the echo level, five estimators are compared:

(a) $e_{\operatorname{Max}}$ is the maximal value of the measured echo level. Hence it concerns only one resolution cell. This is the most instinctive estimator;

(b) $e_{A}$ is the mean echo level in the entire volume. It considers every echo level values of cells with the same weight;

(c) $e_{W}$ represents the weighted arithmetic mean of the distributed echo level given by:

$$
e_{W}=\sum_{n=0}^{M-1} W_{n} \cdot P_{n}
$$

where $P_{n}$ corresponds to a sliding threshold, $W_{n}$ the percentage number of cells for which magnitude $A$ is such as $P_{n+1} \geq A \geq P_{n}, M$ is an integer such as $P_{M}=0 \mathrm{~dB}$ (or the maximal reachable value of echo level). As mentioned before, the echo level step is $P_{n+l}-P_{n}=1 \mathrm{~dB}$. Cells with higher values are assigned to higher weights;

(d) $e_{\mu}$ is the location parameter of the log-normal distribution. It is defined such as:

$$
e_{\mu}=\exp (\mu)
$$

It represents the shift of the log-normal distribution;

(e) $e_{\text {mode }}$ is the mode (maximum point or value of the peak) of the log-normal distribution. It is defined as:

$$
e_{\text {mode }}=\exp \left(\mu-\sigma^{2}\right)
$$

Value indicators of these estimators are plotted on Fig.4.a.

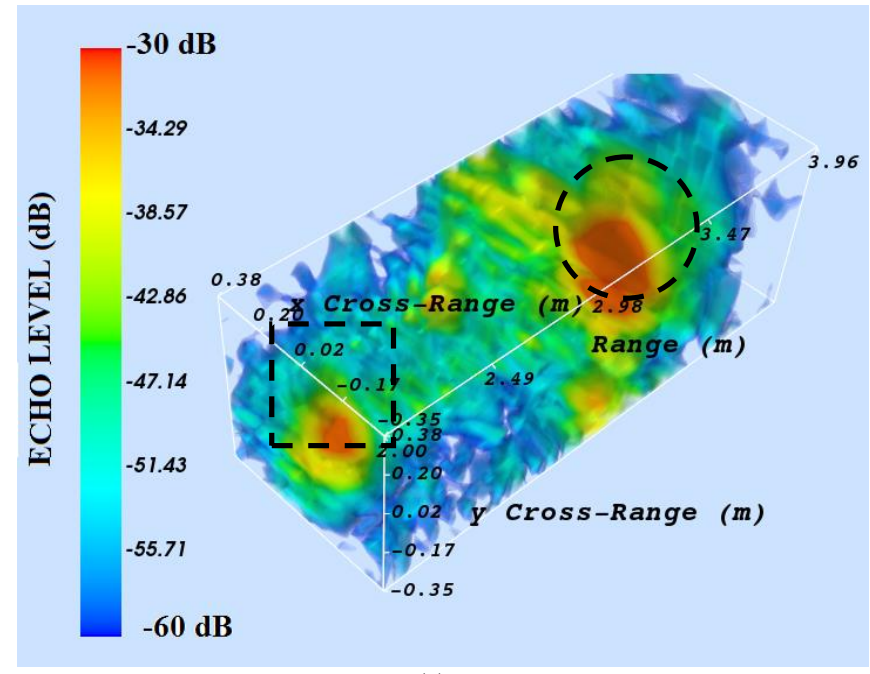

(a)

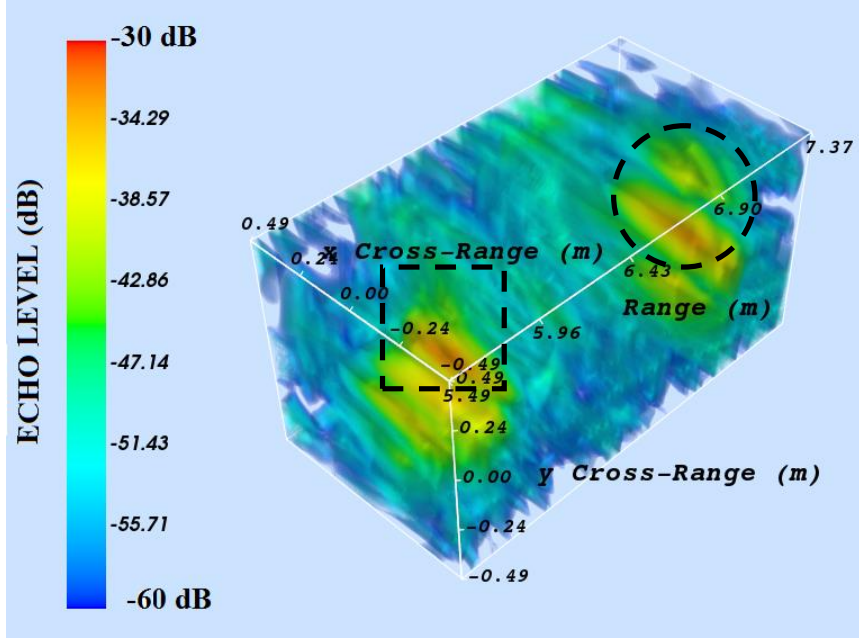

(b)

\begin{tabular}{|c|c|c|c|}
\hline \multicolumn{4}{|c|}{ SENSING MODE VOLUME CHARACTERISTICS } \\
\hline Minimal range & $R_{\text {Min }}$ & $3.20 \mathrm{~m}$ & $6.84 \mathrm{~m}$ \\
\hline Maximal range & $R_{\operatorname{Max}}$ & $4.00 \mathrm{~m}$ & $7.18 \mathrm{~m}$ \\
\hline $\begin{array}{l}\text { x-resolution scale } \\
\text { factor }\end{array}$ & $p_{x}$ & 3.2 & 6.84 \\
\hline $\begin{array}{l}\text { y-resolution scale } \\
\text { factor }\end{array}$ & $p_{y}$ & 3.2 & 6.84 \\
\hline Pad factor & $p$ & 10 & 10 \\
\hline $\mathrm{x}$-axis resolution & $\Delta x$ & $3.5 \mathrm{~cm}$ & $3.5 \mathrm{~cm}$ \\
\hline $\mathrm{y}$-axis resolution & $\Delta y$ & $3.5 \mathrm{~cm}$ & $3.5 \mathrm{~cm}$ \\
\hline z-axis resolution & $\Delta z$ & $7.5 \mathrm{~mm}$ & $7.5 \mathrm{~mm}$ \\
\hline $\mathrm{x}$ cross-range size & $\begin{array}{l}{\left[X_{\text {Min }^{-}}\right.} \\
\left.X_{\text {Max }}\right]\end{array}$ & $\begin{array}{c}{[-31 \mathrm{~cm}, 31} \\
\mathrm{cm}]\end{array}$ & $\begin{array}{c}{\left[\begin{array}{c}-59 \mathrm{~cm}, 31 \\
\mathrm{~cm}]\end{array}\right.}\end{array}$ \\
\hline y cross-range size & $\begin{array}{l}{\left[Y_{M^{-}}\right.} \\
\left.Y_{M a x}\right]\end{array}$ & $\begin{array}{c}{[-38 \mathrm{~cm}, 21} \\
\mathrm{cm}]\end{array}$ & $\begin{array}{c}{[-31 \mathrm{~cm}, 31} \\
\mathrm{cm}]\end{array}$ \\
\hline Resolution cell volume & $V_{\text {Cart }}$ & $9 \mathrm{~cm}^{3}$ & $9 \mathrm{~cm}^{3}$ \\
\hline Total number of cells & $N$ & 32832 & 23598 \\
\hline
\end{tabular}

Fig 3. Structural (square) and sensing (circle) modes representation with isosurfaces for a sensor position at (a) $2 \mathrm{~m}$ and (b) $5.5 \mathrm{~m}$. TABLE I 


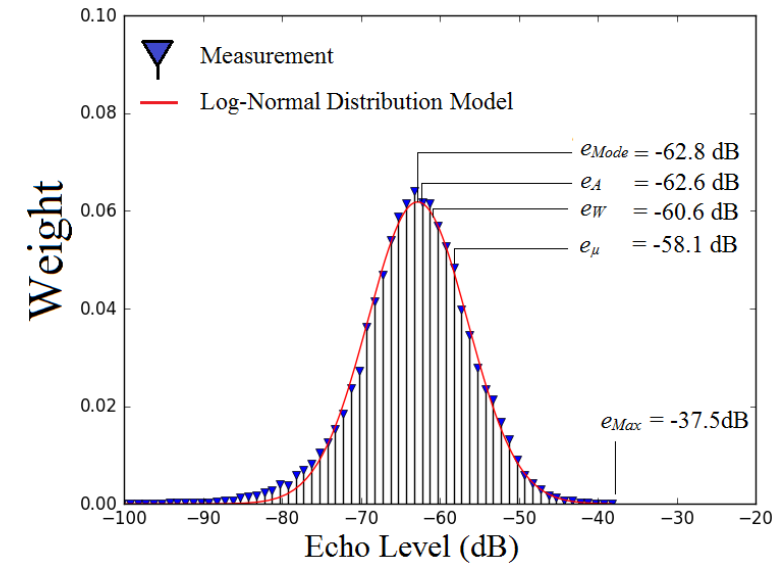

(a)

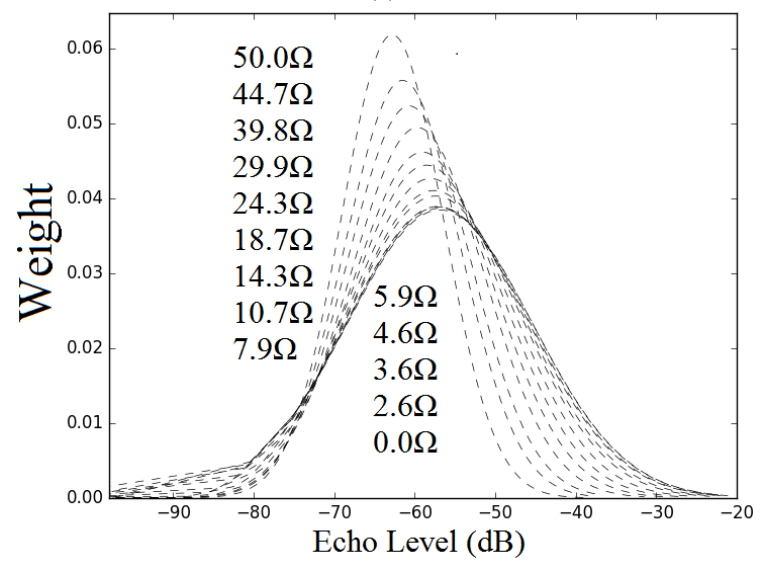

(b)

Fig 4. Distribution of the echo level:

(a) for a sensor input impedance of $50 \Omega$ and a log-normal model (red curve)

(b) log-normal model distribution for multiple sensor input impedances

Estimators are plotted on Fig.5 for the scenario I and scenario II. The estimator $e_{\text {Max }}$ has the best performances in terms of the full-scale measurement range $(17.1 \mathrm{~dB}$ and $15.6 \mathrm{~dB})$ with a good sensitivity $(>-0.4 \mathrm{~dB} / \Omega)$. However there is minimal threshold starting from $40 \Omega$ where the sensor impedance cannot be estimated. That is why the four other estimators are helpful: they take into account the magnitude fluctuation of surrounding cells. Best results are obtained with the estimator $e_{\mu}$ (location parameter of the log-normal distribution). It has full-scale measurement range of $16.5 \mathrm{~dB}$ at $2.0 \mathrm{~m}$ and $10.8 \mathrm{~dB}$ at $5.5 \mathrm{~m}$ with a sensitivity of $-0.3 \mathrm{~dB} / \Omega$. Moreover, it has a linear behavior with a correlation coefficient $\mathrm{R}^{2}>0.990$.

\section{CONCLUSION}

A novel scanning technique has been described in order to remotely read passive sensors. After a brief explanation of the Cartesian grid construction, a display with isosurfaces allows a representation of structural and sensing modes in 3D radar images. Then a study on a specific volume centered on the sensing mode is applied for two sensors positions $(2.0 \mathrm{~m}$ and $5.5 \mathrm{~m}$ ) and for various sensor impedances (from $0 \Omega$ to $50 \Omega$ ). It is observed that the echo level distribution can be modeled as a log-normal distribution and specific estimators are defined to derive the sensor impedance variation for radar images. Estimators that take into account several resolution cells show better results in linearity than one (more intuitive) which considers only the value of the maximal echo level.

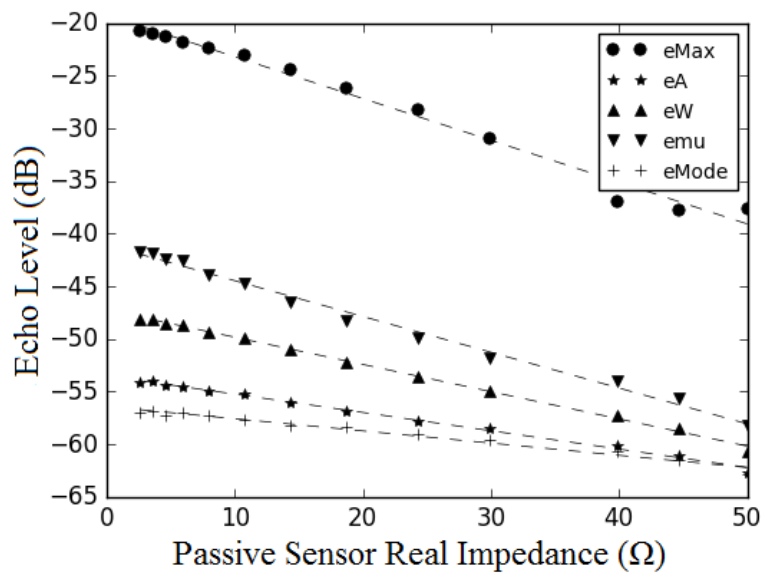

(a)

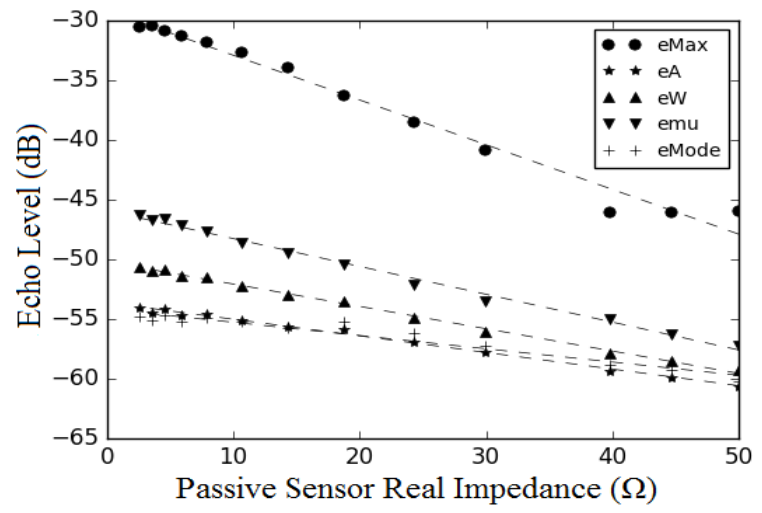

(b)

Fig 5. Echo Level vs. the sensor input resistance using the 5 studied estimators at (a) $2.0 \mathrm{~m}$ and (b) $5.5 \mathrm{~m}$

\section{REFERENCES}

[1] Henry, D.; Pons, P.; Aubert, H., "3D scanning radar for the remote reading of passive electromagnetic sensors," in Microwave Symposium (IMS), 2015 IEEE MTT-S International, pp.1-4, 17-22 May 2015

[2] Henry, D.; Pons, P.; Aubert, H., "3D microwave imaging system for the remote detection and reading of passive sensors," in Microwave Conference (EuMC), 2015 European , pp.259-262, 7-10 Sept. 2015.

[3] S.O. Piper, "Receiver frequency resolution for range resolution in homodyne FMCW radar," Telesystems Conference: Commercial Applications and Dual-Use Technology, 16-17 June 1993, pp.169-173

[4] Harrington, Roger F., "Theory of loaded scatterers," Proceedings of the Institution of Electrical Engineers, vol.111, no.4, pp.617-623, April 1964

[5] Haderer, A.; Scherz, P.; Schrattenecker, J.; Stelzer, A., "Real-time implementation of an FMCW backprojection algorithm for 1D and 2D apertures," in Radar Conference (EuRAD), 2011 European , pp.53-56, 12-14 Oct. 2011.

[6] Rabiner, L.; Schafer, R.W.; Rader, C.M., "The chirp z-transform algorithm," in IEEE Transactions on Audio and Electroacoustics, vol.17, no.2, pp.86-92, Jun 1969 